\title{
Adaptation of Gut Microbiotas to Transgenic Pigs Secreting $\beta$-Glucanase, Xylanase and Phytase
}

Jianxin Mo

Wens Foodstuff Group Co., Ltd

Guoling Li

South China Agricultural University

Guangyan Huang

South China Agricultural University

Haoqiang Wang

OrthoD Group Ltd

Junsong Shi

Wens Foodstuff Group Co., Ltd

Rong Zhou

Wens Foodstuff Group Co., Ltd

Gengyuan Cai

South China Agricultural University

Huaqiang Yang

South China Agricultural University

Zhenfang Wu

South China Agricultural University

Xianwei Zhang (D 15914225968@163.com)

South China Agricultural University https://orcid.org/0000-0001-6205-3050

\section{Research}

Keywords: Transgenic pigs, Microbial enzymes, Gut microbiota, Metagenomics, Feed efficiency

Posted Date: October 7th, 2020

DOl: https://doi.org/10.21203/rs.3.rs-84926/v1

License: (9) This work is licensed under a Creative Commons Attribution 4.0 International License. Read Full License 


\section{Abstract}

\section{Background}

The gut microbiotas play an important role in digestive function and feed efficiency in pigs. However, the effect of exogenous digestive enzymes on the composition and functional contributions of swine intestinal microbes is unclear. The objective of this study was to investigate the change of gut microbiotas in the transgenic pigs secreting microbial digestive enzymes in their salivary glands.

\section{Methods}

EGFP marker-free transgenic (MF-TG) pigs were generated by deleted the EGFP coding genes in the transgenic pigs we previously generated. Samples of chyme from the ileum, caecum and colon of five MF-TG and five wild-type (WT) sows were collected for investigating the gut microbiomes via metagenomics analyses.

\section{Results}

The levels of probiotics were abundant in the caecum of MF-TG pigs and higher than those of WT pigs. By contrast, the levels of some harmful microorganisms were higher in the caecum of WT pigs than those of MF-TG pigs. In addition, the microorganisms in the colon of MF-TG pigs had high fractional abundance in DNA (cytosine-5)-methyltransferase 1 and serine-type D-Ala-D-Ala carboxypeptidase, whereas the aspartate carbamoyltransferase regulatory subunit and outer membrane protein pathways were enriched in WT pigs. Moreover, the levels of numerous carbohydrases in the caecum of MF-TG pigs were higher than those of WT pigs.

\section{Conclusions}

The results indicated that intestinal microbes can change adaptively to the secretion of transgenic enzymes, thereby forming a benign cooperation with their host.

\section{Background}

Livestock industries pursue maximum animal growth rate to utilise the animals' full genetic potential. However, anti-nutrient factors, such as non-starch polysaccharides (NSPs) and phytates, adversely affect feed efficiency, resulting in inefficient feed digestion and substantial rates of nutrient leaching into the environment ${ }^{[1]}$. Pigs produce considerable amounts of nitrogen $(N)$ and phosphorus $(P)$ because they are inherently incapable of digesting NSPs and phytates, which are present in feed grain ${ }^{[2]}$. Various methods have been developed to improve pig nutrient utilisation ${ }^{[3]}$. Dietary supplements with phytate- or NSPdegrading enzymes can effectively reduce $\mathrm{P}$ and $\mathrm{N}$ emissions and improve feed utilisation. However, the effects of supplements are limited by feed production processes, enzyme activity stability and cost. Genetically engineered pigs expressing phytate- or NSP-degrading enzymes in their salivary glands offer 
an alternative and useful strategy for dietary supplementation. Golovan et al. (2001) ${ }^{[2]}$ reported that transgenic (TG) pigs that produce salivary phytase can remarkably improve $P$ digestion from soybean meals. Several other researchers also successfully generated these kinds of TG pigs ${ }^{[4]}$. In our previous studies, we successfully generated TG pigs expressing three microbial enzymes, namely, $\beta$-glucanase, xylanase and phytase, in their salivary glands. These enzymes considerably enhanced the digestion of NSPs and phytates in feedstuff ${ }^{[1]}$. However, these TG pigs also systemically express the EGFP maker, which may have negative effects on pig health and food safety ${ }^{[5]}$.

The mammalian gut microbiotas play critical roles in normal digestive functions, nutrient utilisation, antibiotic resistance and defence against pathogens ${ }^{[6]}$. Dyspeptic gut microbiotas are associated with several intestinal and extraintestinal diseases and poor animal growth performance. These conditions increase the risk of food safety and public health hazards and result in low profitability of animal production ${ }^{[7]}$. Therefore, intestinal microbiotas and their interactions with animal hosts have long been a notable research interest. Although previous studies adopted various animal models, including TG and knock out (KO) animals, to explore the relationship of host genes and the functions of gut microbiotas, none of them directly focused on feed efficiency ${ }^{[8]}$. Thus, the question of whether the endogenous phytate- or NSP-degrading enzymes (phytase, $\beta$-glucanase and xylanase) of TG pigs would alter the composition and activity of intestinal microbiotas remains unanswered. In the present study, we successfully produced EGFP marker-free transgenic (MF-TG) pigs by deleting the EGFP-coding gene in our previously generated TG pigs via the cyclisation recombination enzyme (Cre)-LoxP recombination system. The expression levels of phytase, $\beta$-glucanase and xylanase in MF-TG pigs were similar to those in our previously generated TG pigs. We further tested whether the MF-TG pigs would affect the functional contributions and biological roles of intestinal microbes via metagenomics analyses.

\section{Materials And Methods}

\section{Deletion of the EGFP marker by cyclisation recombination enzyme}

The TG pigs we raised in a previous study were mated with WT sows and then slaughtered on day 30 . The foetuses in the womb were removed, and primary pig foetal fibroblasts (PFFs) were isolated by adherence. The EGFP-tag was deleted by cyclisation recombination enzyme (Excellgen, USA). The PFFs were cultured for another $24 \mathrm{~h}$. Half of the cells were placed into a new 24-well plate. The steps above were repeated twice until the cells grew into a monoclone.

\section{Generation of cloned pigs by somatic cell nuclear transfer}

The marker-free PFFs were used as nuclear donors for SCNT, and the embryos were cultured in vitro overnight. Afterwards, the embryos were transferred to the oviducts of recipient sows. Antibiotics were injected for four consecutive days to reduce inflammation, and the physiological conditions of the recipient sows were recorded daily. In addition, 1000 IU of PMSG was injected into the recipient sows on 
the 10th day after embryo transfer, and 800 IU of hCG was injected on the 13th day to maintain pregnancy.

\section{PCR and Southern blot analyses of founder pigs}

Genomic DNA of founder (F0) cloned pigs were isolated using a DNA tissue kit (OMEGA, USA) according to the manufacturer's protocol. TG and KO genes were amplified via PCR. The PCR products were analysed by $1.5 \%$ agarose gel. For Southern blot, $20 \mu \mathrm{g}$ of genomic DNA was digested and then analysed by $0.8 \%$ agarose gel at $30 \mathrm{~V}$ for $16 \mathrm{~h}$. Subsequently, the gel was washed with alkaline solution, neutralisation solution and $20 \times$ SSC solution and then transferred onto nylon membranes. Genomic DNA was hybridised with digoxigenin-labelled DNA probe. After hybridisation, the nylon membranes were washed and detected with a buffer by using a DIG-high prime DNA labelling and detection starter kit II (Roche, Germany) following the manufacturer's instructions. Finally, the membranes were imaged using the UVP software.

\section{Enzymatic activity assay and Western blot analysis}

Saliva samples of adult MF-TG pigs were collected using non-fat cotton balls. A portion of the samples was tested for enzymatic activity assay at the optimal $\mathrm{pH}$. The other portion was centrifuged using Amicon Ultra-15 (Millipore, USA) for Western blot detection. In brief, $20 \mu \mathrm{g}$ of total protein was subjected to SDS polyacrylamide gel and then transferred onto PVDF membranes (Millipore, USA). The membranes were blocked with $5 \%$ non-fat dry milk for $2 \mathrm{~h}$ and then incubated overnight at $4{ }^{\circ} \mathrm{C}$ with the primary antibodies HA (ab137838, Abcam) or GAPDH (ab8245, Abcam). The membranes were thoroughly washed and then further incubated with a secondary antibody for $2 \mathrm{~h}$ at room temperature. Finally, the membranes were imaged using the UVP software.

\section{Recording of production performance}

The MF-TG pigs (F0) were crossed with WT Duroc sows, and F1 generation MF-TG pigs were propagated and grown until they reached $30 \mathrm{~kg}$ in weight. A total of 14 MF-TG boars $(36.93 \pm 4.77 \mathrm{~kg})$ and $11 \mathrm{WT}$ littermate boars $(39.6 \pm 9.57 \mathrm{~kg})$ were divided into two measuring stations. Ten MF-TG gilts $(34.71 \pm$ $6.06 \mathrm{~kg})$ and $10 \mathrm{WT}$ littermate gilts $(37.88 \pm 5.97 \mathrm{~kg})$ were allocated to three pens according to their initial weight; among them, six MF-TG gilts and eight WT littermate gilts were raised in individual pens. All pigs were allowed free access to water and fed with the same experimental diet formulations made according to the Nutrient Requirements of Swine (NRC, 2012) (Table S4). The experimental formula was designed with $2 \%$ protein reduction, no mineral $\mathrm{P}$ additives and low energy level to evaluate the functional efficiency of the MF-TG pigs. During the experiment, production performance was recorded using MK3 Fire feeders (Fire, USA). In addition, a total of 11 WT boars and 11 gilts were separately tested and fed with complete formula feed as the control (Table S5). Fresh dung samples of the pigs were randomly collected every morning towards the end of the last five days of the trial. The dung samples were stored in a refrigerator at $4{ }^{\circ} \mathrm{C}$. Finally, the samples were mixed well and dried at $80{ }^{\circ} \mathrm{C}$.

\section{Dietary treatment experiments}


Fresh pig manure was collected at 08:00 and 17:00 for five consecutive days. Multipoint collection was adopted with consistent sampling quantity of each point. Manure samples were frozen immediately at $20^{\circ} \mathrm{C}$. Afterwards, the manure of each group was uniformly and thoroughly mixed. Sampling was performed according to the quartile method. Total nitrogen was measured using fresh dung samples. The remaining samples were dried at $80^{\circ} \mathrm{C}$. The samples were air-dried and then crushed to particles < $0.425 \mathrm{~mm}$ in size for $\mathrm{Ca}$ and $\mathrm{P}$ content measurements.

\section{Metagenomics sequencing and statistical analyses}

Five MF-TG sows $(115.76 \pm 1.57 \mathrm{~kg})$ and five WT sows $(116.04 \pm 0.55 \mathrm{~kg})$ were selected for metagenomics analyses. During the fattening stage, the pigs were raised with the same experimental diet in human-controlled farm conditions and similar management schemes. The intestinal contents were collected from the ileum, caecum and colon and then immediately transferred to liquid nitrogen for temporary storage. The genomic DNA of the samples was then isolated and used to construct a sequencing library. The libraries were generated using the DNA library prep kit for Illumina (NEB, USA) and the raw data obtained from the Illumina HiSeq sequencing platform.

Scaftigs ( $\geq 500 \mathrm{bp}$ ) assembled from both single and mixed samples were all predicted the ORFs by using the MetaGeneMark software. ORFs with length shorter than $100 \mathrm{nt}$ was filtered from the predicted result with default parameters. For ORF prediction, the CD-HIT software was used to remove redundancy and obtain unique initial gene catalogues. Clean data of each sample were mapped onto an initial gene catalogue by using Bowtie2.2.4. The number of reads to which genes mapped in each sample was obtained with the parameter settings end-to-end, sensitive, I 200 and X 400. In each sample, the gene with $\leq 2$ number of reads was filtered to obtain the gene catalogue (unigenes) for subsequent analysis. On the basis of the number of mapped reads and gene lengths, the abundance information of each gene in each sample was analysed.

The DIAMOND software was used to BLAST the unigenes to the sequences of bacteria, fungi, archaea and viruses, which were extracted from the NR database of NCBI with the parameter settings blastp -e 1e5. For the aligned results of each sequence, as each sequence may have multiple aligned results, the result with the e value $\leq$ the smallest e value * 10 was selected. The LCA algorithm was applied to the system classification of the MEGAN software to ensure the species annotation information of the sequences. A table containing the number of genes and the abundance information of each sample in each taxonomic hierarchy was constructed on the basis of LCA annotation results and the gene abundance table. LEfSe analysis was performed to determine differences in species composition among groups. Permutation test between groups was used for Metastats analysis of each taxonomic group and to obtain the $P$ value. Benjamini and Hochberg False Discovery Rate was utilised to correct the $P$ value and acquire the q value. LEfSe analysis was conducted using the LEfSe software. Finally, random forest was implemented to construct a random forest model. Important species were screened by MeanDecreaseAccuracy and MeanDecreaseGin. 
The DIAMOND software was used to BLAST the unigenes to the functional database with the parameter settings blastp, -e 1e-5. The functional databases included the KEGG, eggNOG and CAZy databases. For each sequence's BLAST result, the best BLAST Hit was utilised for subsequent analysis. The relative abundance of different functional hierarchies was analysed. In this study, the relative abundance of each functional hierarchy was equal to the sum of relative abundance annotated to that functional level. On the basis of functional annotation results and the gene abundance table, the gene number table of each sample in each taxonomic hierarchy was obtained. The gene number of a function in a sample was equal to the gene number annotated to this function and the abundance was nonzero.

For resistance gene annotation, the RGI software was employed to align the unigenes to the CARD database with the parameter settings blastp, evalue $\leq 1 \mathrm{e}-30$. On the basis of the aligned results, the relative abundance of ARO was counted. On the basis of the abundance of ARO, abundance bar charts and abundance cluster heat maps were created, and differences in the number of resistance genes between groups were determined. Furthermore, analyses of the abundance distribution of the resistance genes in each sample, species attribution of resistance genes and resistance mechanism of resistance genes were conducted.

\section{Statistical analyses}

The data were analysed by the GLM procedure (SAS, USA). For growth performance, covariance analysis was performed, and initial body weight and experimental period were used as the covariates. For apparent faecal nutrient emission, one-way ANOVA followed by Duncan's multiple comparison were conducted. Unpaired two-sample $t$-test (two-tailed test) was used for enzymatic activity analyses. Statistical significance was set to $P<0.05$.

\section{Results}

\section{Generation of MF-TG pigs}

The EGFP-tag was deleted by Cre, and the MF-TG pigs were generated by somatic cell nuclear transfer (SCNT). The primers of PCR and Southern blot were used to identify TG fragments (Fig. 1A). A total of eight cloned piglets were born, all of which were MF-TG pigs according to PCR and Southern blot analyses (Figs. 1B and 1C). Different tissues and organs of the MF-TG pigs were collected. Western blot revealed that the MF-TG pigs efficiently expressed $\beta$-glucanase, xylanase and phytase only in the parotid gland and not in other tissues (Fig. 1D). During the feeding period, we collected saliva samples of 6month-old MF-TG and WT pigs for enzymatic activity assays. The assays detected enzymatic activity in all MF-TG pigs, among which 903 had the highest enzyme activity; 2.5, 0.98 and $2.07 \mathrm{U} / \mathrm{mL}$ of $\beta$ glucanase, xylanase and phytase was detected, respectively (Fig. 1. E).

\section{MF-TG pigs had improved feed utilisation and reduced nutrient emission}


MF-TG boars (803, 903 and 907) were crossed with WT Duroc sows. 48 offspring were born, of which 24 were MF-TG pigs and 24 were WT littermate pigs. A total of 24 MF-TG pigs ( 14 boars and 10 gilts) and 21 WT littermates (11 boars and 10 gilts) were raised together and fed with nutrition-deficient experimental diets to measure the growth performance of the MF-TG pigs (Table S4). Although the difference in average daily feed intake (ADFI) between MF-TG and WT pigs was not significant $(P=0.95$ and 0.05 for male and female, respectively), the MF-TG pigs had better feed consumption, higher average daily gain $(A D G)$, better feed conversion rate (FCR) and shorter day to market. Feed consumption decreased by $11.85 \mathrm{~kg}$ to $21.57 \mathrm{~kg}, \mathrm{ADG}$ increased by $131.34 \mathrm{~g}$ to $162.88 \mathrm{~g}$, FCR declined by 0.18 to 0.42 , and day to market shortened by 9.42 days to 15.07 days (Table 1). We further investigated the effects of the microbial enzymes secreted by MF-TG pigs on nutrient emission. Results showed that the P emission of MF-TG pigs significantly decreased by about $26.45-26.52 \%$, but their $\mathrm{N}$ and Ca excretion did not significantly change compared with that of the WT1 group. Compared with WT2 boars fed with special diets for breeding pigs (Table S11), the contents of faecal N, P and Ca of MF-TG boars decreased by $17.10 \%, 51.95 \%$ and $72.65 \%$, respectively (Fig. $2 A$ ) and those of MF-TG gilts declined by $15.10 \%, 52.42 \%$ and $67.15 \%$, respectively (Fig. 2B). The MF-TG pigs were as good as or even better than the WT2 control group in terms of growth performance (Table S12).

\section{Comparison of the gut microbial communities of MF-TG and WT sows}

We collected samples from the ileum, caecum and colon of five MF-TG sows and five WT sows and investigated their gut microbiotas via metagenomics analyses. A total of $1,832,628$ predicted genes (71.26\%) were annotated into the NR database, and their proportions at the kingdom, phylum, class, order, family, genus and species levels were $82.85 \%, 79.04 \%, 72.80 \%, 72.25 \%, 60.07 \%, 55.22 \%$ and $42.53 \%$, respectively (Table S5). We selected the top 10 microorganisms with the largest relative abundance in each sample and integrated the clustering results with the relative abundance at the phylum level by using the Bray-Curtis distance for cluster analysis. At the phylum level, the abundance of ileal (IL) microorganisms was substantially lower than that of caecal (Ce) and colonic (Co) microorganisms. Nevertheless, individual samples were highly variable. The main $\mathrm{Ce}$ and Co microorganisms were Firmicutes, Bacteroidetes and Euryarchaeota, whereas the primary IL microorganisms were Proteobacteria and Firmicutes. Furthermore, the difference in the microbiotas of the same intestinal parts of MF-TG pigs and WT pigs was not significant (Fig. 3A and Table S6). Principal component analysis (PCA) revealed that the distance between IL and Co/Ce was far and clustered into a different category, but $\mathrm{Co}$ and Ce could not be separated (Fig. 3B and Table S7). We analysed the differences in species composition among different groups via LEfSe analysis. We evaluated the abundance of different species by using LDA scores. Results showed that the WT pigs had significantly higher proportions of harmful microorganisms, including Campylobacter (causes diarrhoea ${ }^{[9]}$, Chlamydia trachomatis (associated with pneumonia ${ }^{[10]}$, C. fetus (causes septicaemia ${ }^{[11]}$ ) and various unidentified viruses, than the MF-TG pigs. By contrast, the MF-TG pigs had higher levels of probiotics, such as Lactobacillus reuteri and Streptococcus, in caecum than the WT pigs (Fig. 3C). 


\section{Comparison of functions and abundance of microbial genes of MF-TG and WT pigs}

For resistance gene annotation, unigenes were compared with the CARD database by using the resistance gene identifier (RGI) software. Results showed that the difference in the abundance of resistance genes of MF-TG and WT pigs was not significant (Fig. 4A and Table S8). Moreover, common veterinary drugs corresponded with resistance genes in the pig intestines, such as tetracycline resistance protein, aminoglycoside antibiotic kinase, lincomycin resistance, tetracycline efflux gene, erythromycin resistance and flornicol resistance gene. The abundance of Fox-5, a cephalosporin resistance gene, was the highest in the ileum, whereas tetW was the highest in the colon and caecum (Fig. 4B and Table S9). We analysed the different KEGG functions of MF-TG and WT pigs. Results showed that the microorganisms in the colon of MF-TG pigs had high fractional abundance in DNA-methyltransferase 1 (K00558, cysteine and methionine metabolism) and serine-type D-Ala-D-Ala carboxypeptidase (K07258, peptidoglycan biosynthesis), whereas the aspartate carbamoyltransferase regulatory subunit (K00610, nucleotide metabolism) and outer membrane protein (K06142, signalling and cellular processes) pathways were enriched in WT pigs (Fig. 4C). A comparison of the unigenes and the carbohydrate enzyme (CAZy) database revealed that the gene abundance of transgenic caecal microorganisms was active in $\mathrm{GH} 8$ (cellulase and endo-1,4- $\beta$-xylanase), GH13 (a-amylase, 4- $\alpha$-glucanotransferase) and GH32 (endo-levanase, exo-inulinase) (Fig. 4D). We then analysed the CAZy database by using the DIAMOND software. Results showed that 50 carbohydrate enzymes were significantly enriched in the caecal microorganisms of MFTG pigs, indicating that the MF-TG pigs had a good digestive activity (Fig. 5 and Table S10).

\section{Discussion}

Generation of genetically modified pigs is an efficient strategy for improving various indicators of pig performance, such as feed utilisation, piglet survival rate and pork nutritional composition. In our previous study, we generated a transgenic pigs expressing four microbial enzymes, namely, $\operatorname{bg} 17 \mathrm{~A}$, eg1314, xynB and eappA, in salivary glands specifically ${ }^{[1]}$. During the feeding process, we found that the $\mathrm{N}$ and $\mathrm{P}$ emissions of the TG pigs were substantially reduced, whereas their nutrient intake and absorption from the feed increased. However, the TG pigs carry the EGFP gene. This gene has potential animal and human health hazards ${ }^{[12]}$. In the present study, we developed TG pigs without the EGFP marker. The MF-TG pigs were found to efficiently secrete the microbial enzymes phytase, $\beta$-glucanase and xylanase as the previous TG pigs. We compared the MF-TG pigs with WT pigs fed with complete diets and noted that these genetically modified pigs fed with nutrition-deficient experimental diets considerably reduced their $\mathrm{N}, \mathrm{P}$ and $\mathrm{Ca}$ emissions in the manure without compromising production performance. However, when compared with WT pigs fed with the same nutrition-deficient experimental diets, these genetically modified pigs had substantially improved ADG and feed utilisation efficiency but only reduced faecal $\mathrm{P}$ emissions. This result was not consistent with that of our previous trial in metabolic cage under restrictive feeding and movement ${ }^{[1]}$. The main difference between the previous study and the present work was that all pigs herein were measured in cages without feeding and 
movement restrictions. Other prior studies reported that supplementary enzymes in the feed have a positive effect on the digestibility of feed nutrients ${ }^{[3,13]}$. Phytase has the ability to liberate $P$ from phytate by step-wise dephosphorylation of phytate. $\beta$-glucanase and xylanase can effectively degrade glucan and xylan, respectively. Hence, $\beta$-glucanase, xylanase and phytaseare are both nutritionally and ecologically beneficial because they enhance $\mathrm{P} / \mathrm{N}$ absorption while reducing $\mathrm{P} / \mathrm{N}$ excretion ${ }^{[1]}$.

The issue of whether the digestive enzymes secreted by genetically modified pigs would affect their intestinal microbiotas remains unclear. In recent years, metagenomics methods based on highthroughput sequencing technology have rapidly promoted the study of the composition and function of intestinal microorganism floras ${ }^{[14]}$. In the present study, all experimental pigs were selected from populations with a similar genetic background, of the same gender and raised under the same environmental, nutritional and management conditions to minimise the variability caused by genetic, gender and external factors. Nevertheless, results showed that the gut microbiotas of MF-TG and WT pigs were different, consistent with those of previous studies ${ }^{[15-17]}$. The MF-TG pigs had higher levels of probiotics, such as $L$. reuteri and Streptococcus, in the caecum than WT pigs. Lactobacillus reuteri strongly adheres to the intestinal mucosa; thus, this bacterium can improve the distribution of intestinal microbes, antagonise the colonisation of other harmful bacteria and prevent the development of intestinal diseases ${ }^{[18]}$. In addition, Lactobacillus reuteri can produce reuterin, a non-protein broadspectrum antibacterial substance that can greatly inhibit the growth of Gram-positive/negative bacteria, yeast, fungi and pathogens ${ }^{[18]}$. Streptococcus is generally considered a health-promoting microorganism because of its role in regulating human health. Numerous Streptococcus species are involved in carbohydrate fermentation, starch hydrolysis and glucan production from sucrose ${ }^{[17]}$. Streptococcus gallolyticus can ferment mannitol, trehalose and inulin and produce acids from starch and glycogen. Therefore, the presence of these bacteria suggested that the MTF-TG pigs were healthier than the WT pigs because they have more probiotics to promote gut health or degrade carbohydrates in their diet. By contrast, the levels of Campylobacter and Chlamydia in WT pigs were higher than those in MF-TG pigs. Campylobacter and Chlamydia are common parasites in the digestive tract of numerous livestock, such as cattle, sheep, pig and poultry, and often cause diarrhoea and enteritis ${ }^{\left[{ }^{[-11]}\right.}$. The presence of these parasites indicated that the WT pigs were more susceptible to diarrhoea and enteritis than the MF-TG pigs. Other studies also suggested that the levels of NSP-degrading enzymes tend to increase the population of beneficial bacteria, thereby enhancing gut physiology, for example, reducing the relative weight of organs in the digestive system and increasing villus height ${ }^{[19]}$.

We also compared the functions and abundance of microbial genes of MF-TG and WT pigs. Results showed that the abundance of K07258, K00610 and K06142 in the caecum of MF-TG pigs was more active than that of WT pigs. These genes are associated with cysteine and methionine metabolism, peptidoglycan biosynthesis and nucleotide metabolism. Prior studies reported that pigs with high feed utilisation have high abundance of methionine metabolism, peptidoglycan biosynthesis and nucleotide metabolism pathways. These features seem to verify that the gut microorganisms in MF-TG pigs can adapt to multiple digestive enzymes and evolve new mechanisms to proliferate despite altered metabolic 
conditions $^{[17]}$. In addition, the abundance of 50 carbohydrate enzymes in the caecal microbes of MF-TG pigs was high as expected. These results on the functions of microbial genes indicated that the microorganisms promoted the adaptability of transgenic enzymes and increased the feed efficiency of the MF-TG pigs. Moreover, common veterinary drugs were associated with their corresponding resistance genes in the pig intestine, suggesting that the intestinal microbes acquired resistance under the pressure of antibiotic selection. Nevertheless, the evolution of antibiotic resistance seemed to have no relationship with the MF-TG pigs expressing exogenous digestive enzymes.

\section{Conclusions}

Results from the present study demonstrated that the MF-TG pigs secreting NSP-degrading enzymes and phytase in the salivary glands can greatly promote nutrient absorption, improve growth performance and reduce pollutant emissions. Moreover, the intestinal microbiotas exhibited adaptive changes to the TG enzymes, which are beneficial to animal nutrient utilisation and health.

\section{Declarations}

\section{Acknowledgements}

Not applicable.

\section{Authors' contributions}

The contributions of the authors were as follows: Jianxin Mo conducted the animal work, most of the laboratory work and revise the manuscript. Guoling Li conducted part of the laboratory work and wrote the manuscript. Guangyan Huang, Haoqiang Wang, Junsong Shi, Rong Zhou, Gengyuan Cai and Huaqiang Yang helped to conduct animal trial and part of the laboratory work. Zhenfang Wu and Xianwei Zhang designed the experiment, oversaw the development of the study and wrote the last version of the manuscript. The authors declare no conflicts of interest. The authors read and approved the final manuscript.

\section{Funding}

This work was supported by grants from the National Science and Technology Major Project for Breeding of New Transgenic Organisms (2016ZX08006002) and the Local Innovative and Research Teams Project of Guangdong Province (2019BT02N630).

\section{Availability of data and materials}

All data generated or analyzed during this study are available from the corresponding author upon reasonable request.

\section{Ethics approval}


The protocol for this experiment was reviewed and approved by the Instructive Notions with Respect to Caring for Laboratory Animals issued by the Ministry of Science and Technology of China. This study was approved by the Animal Care and Use Committee of the South China Agricultural University.

\section{Consent for publication}

Not applicable.

\section{Conflict of interest}

The authors declare that they have no competing interests

\section{References}

1. Xianwei Z, Zicong L, Huaqiang Y, Dewu L, Gengyuan C, Guoling L, et al. Novel transgenic pigs with enhanced growth and reduced environmental impact. Elife. 2018;7:e34286-.

2. Golovan SP, Meidinger RG, Ajakaiye A, Cottrill M, Wiederkehr MZ, Barney DJ, et al. Pigs expressing salivary phytase produce low-phosphorus manure. Nature Biotechnology. 2001;19:741-5.

3. Swiatkiewicz S, Swiatkiewicz M, Arczewska-Wlosek A, Jozefiak D. Efficacy of feed enzymes in pig and poultry diets containing distillers dried grains with solubles: a review. J Anim Physiol Anim Nutr. 2016;100:15-26.

4. Lin YS, Yang CC, Hsu CC, Hsu JT, Wu SC, Lin CJ, et al. Establishment of a novel, eco-friendly transgenic pig model using porcine pancreatic amylase promoter-driven fungal cellulase transgenes. Transgenic Research. 2015.

5. Bi Y, Hua Z, Liu X, Hua W, Ren H, Xiao H, et al. Isozygous and selectable marker-free MSTN knockout cloned pigs generated by the combined use of CRISPR/Cas9 and Cre/LoxP. Sci Rep. 2016;6:31729.

6. Sommer F, Bäckhed F. The gut microbiota--masters of host development and physiology. Nat Rev Microbiol. 2013;11:227-38.

7. Wang W, Hu H, Zijlstra RT, Zheng J, Gänzle MG. Metagenomic reconstructions of gut microbial metabolism in weanling pigs. Microbiome. 2019;7.

8. Qingqing W, Lili Q, Shengwang J, Chunbo C, Dezun M, Pengfei G, et al. Safety Evaluation of Neo Transgenic Pigs by Studying Changes in Gut Microbiota Using High-Throughput Sequencing Technology. PLoS ONE. 2016;11:e0150937.

9. Burnham PM, Hendrixson DR. Campylobacter jejuni : collective components promoting a successful enteric lifestyle. Nature Reviews Microbiology. 2018.

10. Lu C, Holland MJ, Gong S, Peng B, Bailey RL, Mabey DW, et al. Genome-Wide Identification of Chlamydia trachomatis Antigens Associated with Trachomatous Trichiasis. Investigative Ophthalmology \& Visual ence. 2012;53.

11. Sachse K, Grossmann E. [Chlamydial diseases of domestic animals--zoonotic potential of the agents and diagnostic issues]. Dtw Deutsche Tierrztliche Wochenschrift. 2002;109:142. 
12. Olesya V. Stepanenko VVV, Irina M. Kuznetsova, Vladimir N. Uversky, K. K. Turoverov. Fluorescent Proteins as Biomarkers and Biosensors: Throwing Color Lights on Molecular and Cellular Processes. Current Protn \& Peptide ence. 2008;9:-.

13. Recharla N, Kim D, Ramani S, Song M, Park J, Balasubramanian B, et al. Dietary multi-enzyme complex improves In Vitro nutrient digestibility and hind gut microbial fermentation of pigs. PLoS ONE. 2019;14:e0217459.

14. Costea PI, Hildebrand F, Manimozhiyan A, Bäckhed F, Bork P. Enterotypes in the landscape of gut microbial community composition. Nat Microbiol. 2018;3:8-16.

15. Nicholson JK, Holmes E, Kinross J, Burcelin R, Gibson G, Jia W, et al. Host-gut microbiota metabolic interactions. Science. 2012;336:1262-7.

16. Parks BW, Nam E, Org E, Kostem E, Norheim F, Hui ST, et al. Genetic control of obesity and gut microbiota composition in response to high-fat, high-sucrose diet in mice. Cell Metab. 2013;17:14152.

17. Quan J, Cai G, Yang M, Zeng Z, Wu Z. Exploring the Fecal Microbial Composition and Metagenomic Functional Capacities Associated With Feed Efficiency in Commercial DLY Pigs. Frontiers in Microbiology. 2019;10:-.

18. Kleerebezem M, Vaughan EE. Probiotic and Gut Lactobacilli and Bifidobacteria: Molecular Approaches to Study Diversity and Activity. Annual Review of Microbiology. 2009;63:269-90.

19. Zijlstra RT, Owusu-Asiedu A, Simmins PH. Future of NSP-degrading enzymes to improve nutrient utilization of co-products and gut health in pigs. Livestock ence. 2010;134:255-7.

\section{Tables}

Due to technical limitations, table 1 is only available as a download in the Supplemental Files section.

\section{Figures}


A

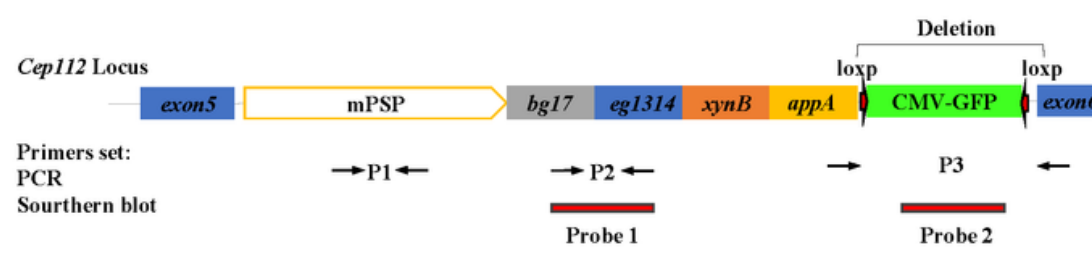

B

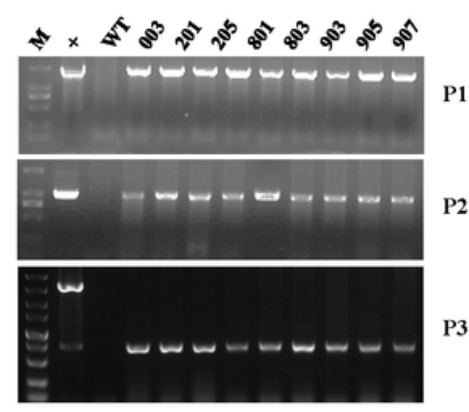

$\mathbf{E}$

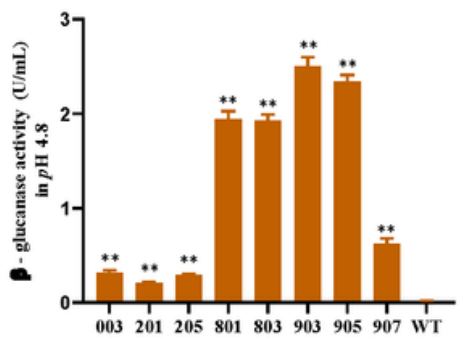

C
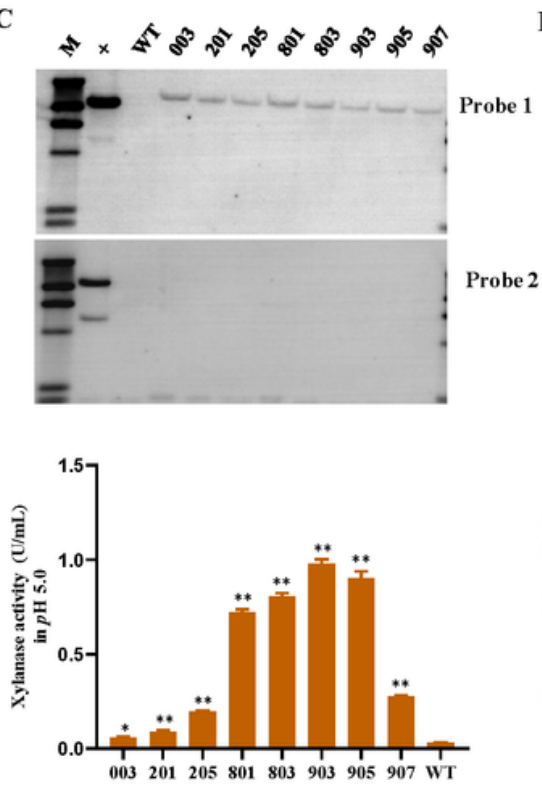

D
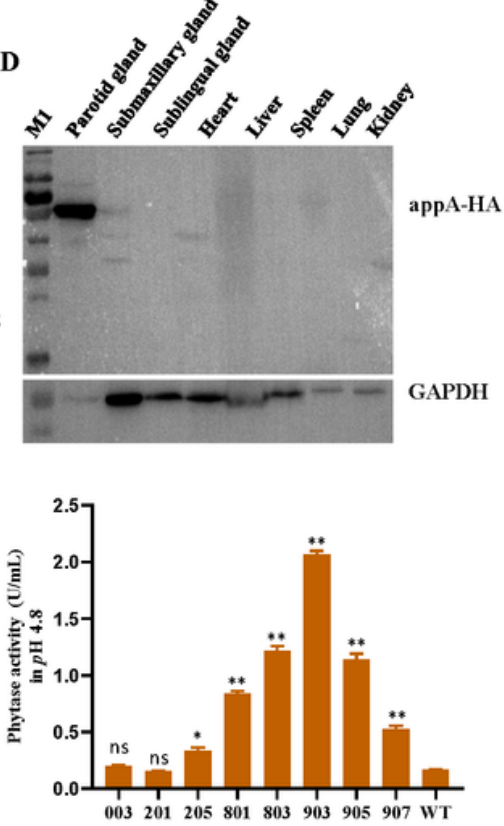

\section{Figure 1}

Characterisation of transgene and expression in founder MF-TG pigs. A) Four microbial enzymes were integrated into porcine CEP112 intron 5 after deleting the EGFP-tag. Different primers (P1, P2 and P3) were used to confirm the occurrence of transgene, Probe 1 and probe 2 were designed for Southern blot analysis. B) Genomic DNA of the cloned pigs was amplified by PCR and analysed by gel electrophoresis. C) Southern blot analysis revealed that multiple enzyme transgenes were integrated into porcine CEP112 intron 5 without the EGFP maker. D) Western blot analysis demonstrated that the microbial enzymes were specifically expressed in the parotid gland of MF-TG pigs. E) MF-TG pigs could efficiently express $\beta$ glucanase, xylanase and phytase in their salivary gland. Values are shown as mean \pm SEM. An asterisk represents $P<0.05$, whereas double asterisks denote $P<0.01$ versus control. 
A

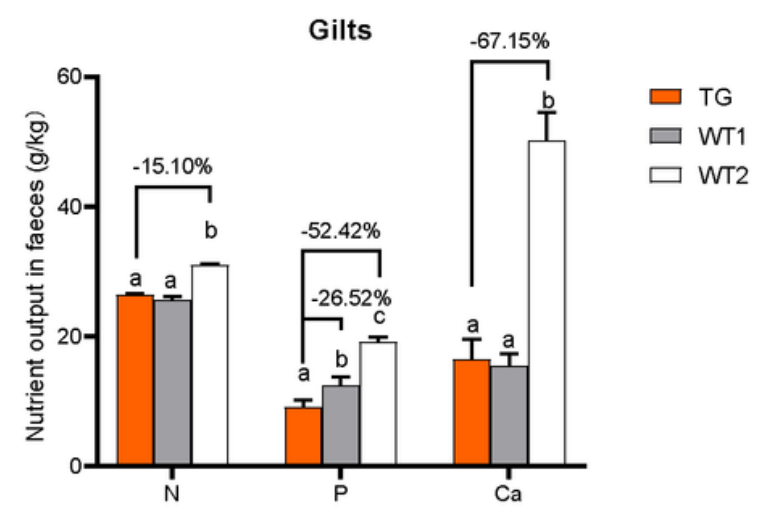

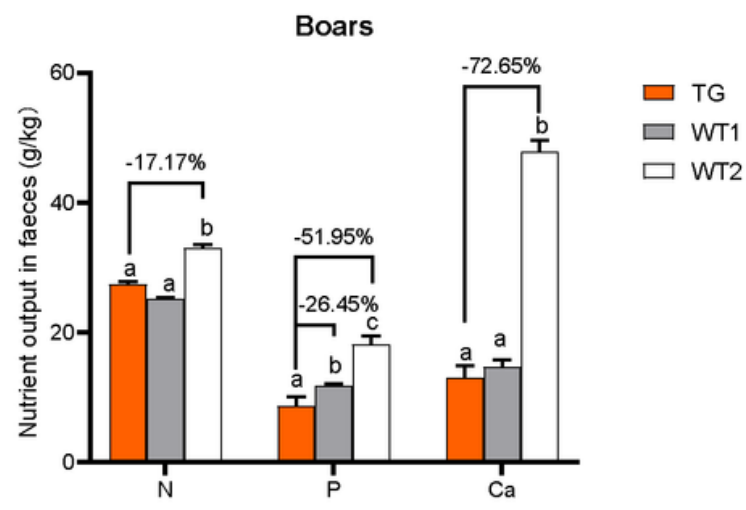

\section{Figure 2}

Comparison of nutrient emissions of MF-TG pigs and WT littermates. Utilisation of faecal calcium (Ca), nitrogen $(N)$ and phosphorus $(P)$ was detected in MF-TG gilts $(A)$ and boars $(B)$. Data are shown as mean \pm SEM. MF-TG $(n=5)$ and WT1 $(n=6)$ pigs were fed with experimental diets with low nitrogen, no mineral phosphorus and low energy level.WT2 pigs $(n=11)$ were fed with commercial diets. Different letters represent $\mathrm{P}<0.05$. 
A

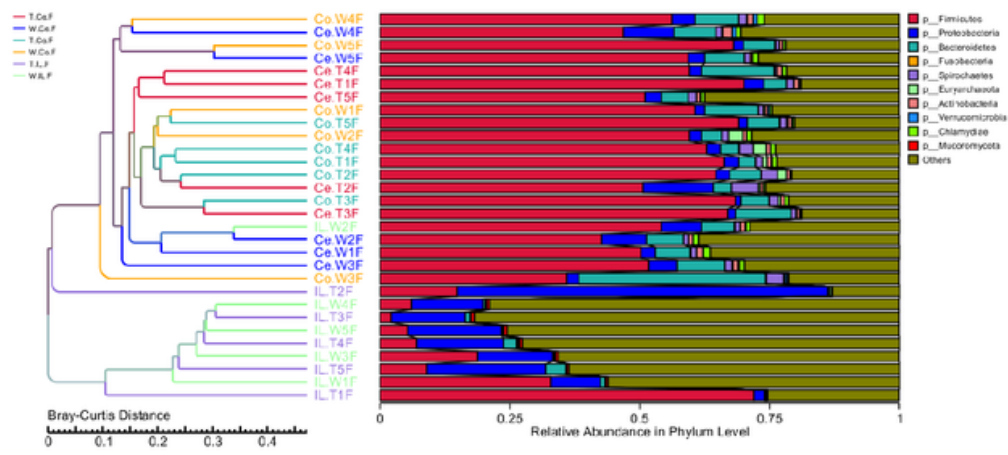

B

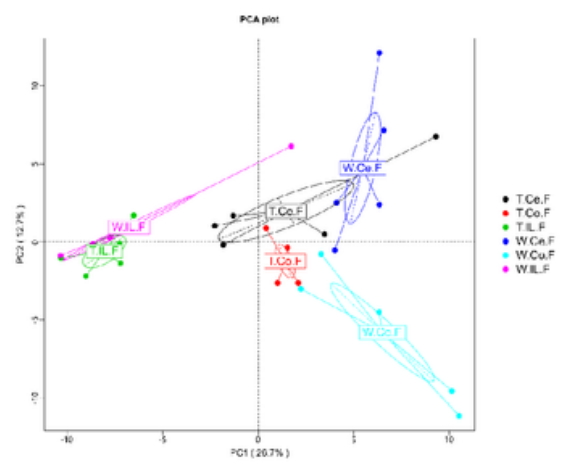

C

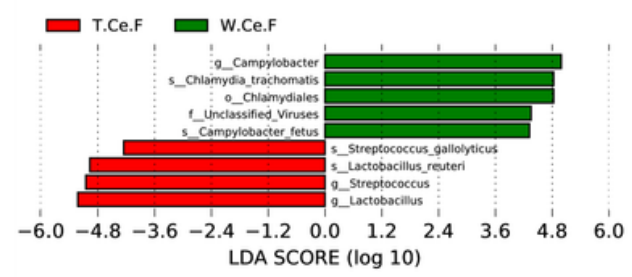

\section{Figure 3}

Comparison of intestinal microbial communities of MF-TG pigs and WT littermates. A) Community composition at the phylum level in MF-TG females and WT littermates. IL, Ce and Co stand for ileum, caecum and colon, respectively. MF-TG and WT female pigs stand for TF and WF, respectively. The BrayCurtis distance was used for cluster analysis of relative abundance at the phylum level to detect similarities in different samples. B) Principal component analysis (PCA) results of the intestinal microbiotas at the phylum level. C) The LDA value distribution of different species shown as a histogram. The length of the histogram represents the relative effect of different species. 
A

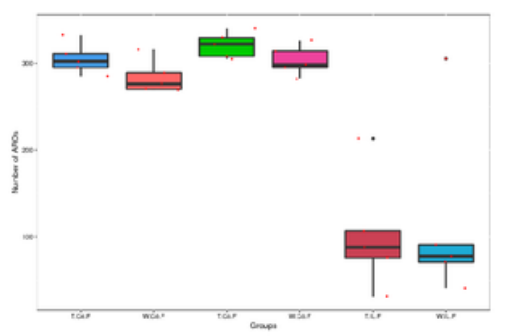

C

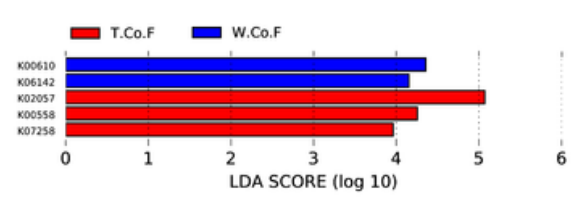

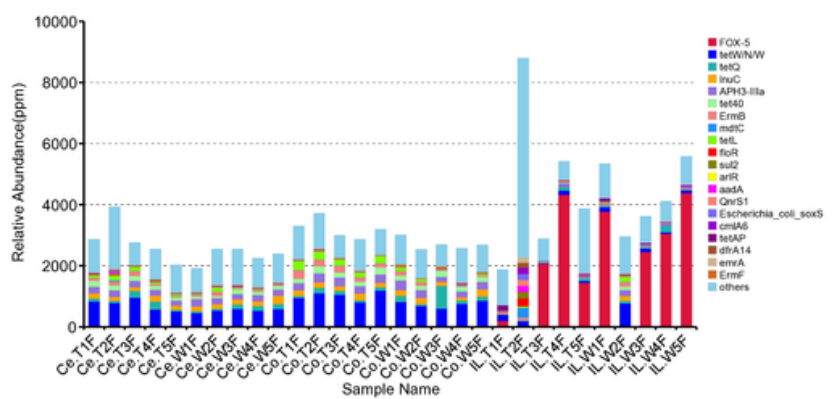

D

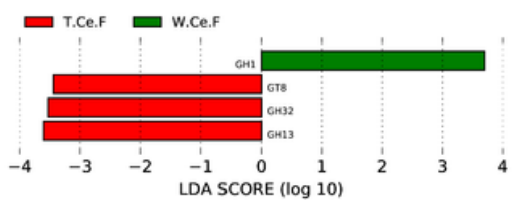

\section{Figure 4}

Functional annotation and abundance of microbial genes in different intestinal microorganisms. A) The number of ARO in MF-TG and WT pigs was not significantly different. B) The relative abundance of the top 20 ARO is shown as a histogram. LEfSe analysis revealed the different functions in KEGG (C) and CAZy (D) databases. E) On the basis of the CAZy-LDA scores, heat maps of different functions were drawn to reflect the distribution of these functions in each sample. 


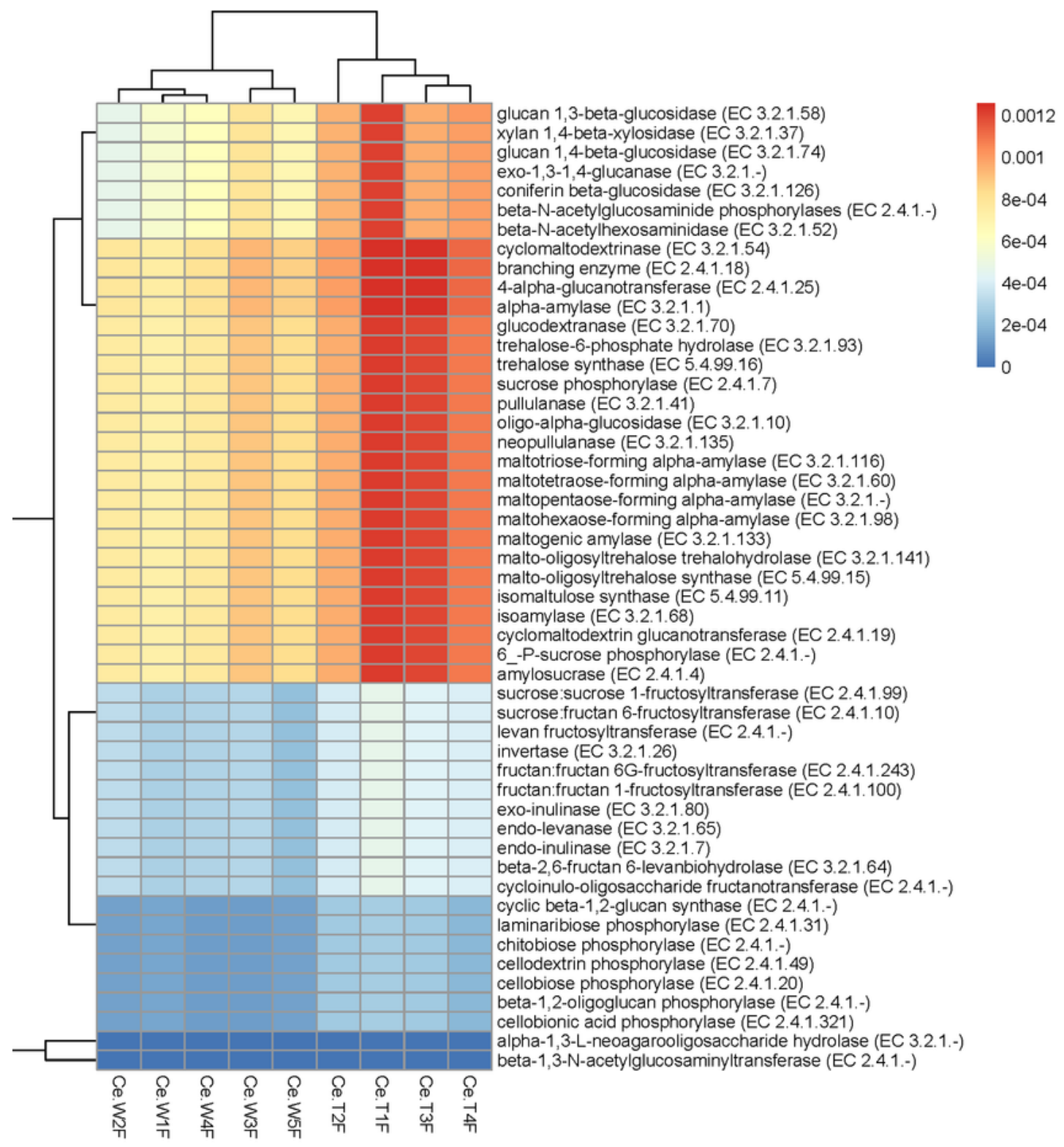

\section{Figure 5}

We then analysed the CAZy database by using the DIAMOND software. Results showed that 50 carbohydrate enzymes were significantly enriched in the caecal microorganisms of MF-TG pigs, indicating that the MF-TG pigs had a good digestive activity

\section{Supplementary Files}


This is a list of supplementary files associated with this preprint. Click to download.

- TableS12.pdf

- Tables9.xlsx

- TableS6.xlsx

- TableS1S5S78S1011.pdf

- Table.1.pdf 